Article

\title{
Probing Structures of Interfacial 1-Butyl-3-Methylimidazolium Trifluoromethanesulfonate Ionic Liquid on Nano-Aluminum Oxide Surfaces Using High-Pressure Infrared Spectroscopy
}

\author{
Hai-Chou Chang ${ }^{1, *} \mathbb{D}$, Teng-Hui Wang ${ }^{1}$ and Christopher M. Burba ${ }^{2}$ \\ 1 Department of Chemistry, National Dong Hwa University, Shoufeng, Hualien 974, Taiwan; \\ 410112029@ems.ndhu.edu.tw \\ 2 Department of Natural Science, Northeastern State University, Tahlequah, OK 74464, USA; \\ burba@nsuok.edu \\ * Correspondence: hcchang@gms.ndhu.edu.tw; Tel.: +886-3-863-3585
}

Received: 20 July 2017; Accepted: 15 August 2017; Published: 18 August 2017

\begin{abstract}
The interactions between 1-butyl-3-methylimidazolium trifluoromethanesulfonate ([BMIM][TFS]) and nano- $\mathrm{Al}_{2} \mathrm{O}_{3}$ are studied using high-pressure infrared spectroscopy. The thickness of the [BMIM][TFS] interfacial layer on the aluminum oxide are adjusted by controlling the number of washes with ethanol. In contrast to the results obtained under ambient pressure, local structures of both the cations and anions of [BMIM][TFS] are disturbed under high pressures. For example, bands due to $\mathrm{C}-\mathrm{H}$ stretching motions display remarkable blue-shifts in frequency as the pressure of the [BMIM][TFS] $/ \mathrm{Al}_{2} \mathrm{O}_{3}$ composites is increased to $0.4 \mathrm{GPa}$. The bands then undergo mild shifts in frequency upon further compression. The discontinuous jump occurring around $0.4 \mathrm{GPa}$ becomes less obvious when the amount of ionic liquid on the $\mathrm{Al}_{2} \mathrm{O}_{3}$ is reduced by washing with ethanol. The nano- $\mathrm{Al}_{2} \mathrm{O}_{3}$ with surfaces may weaken the cation/anion interactions in the interfacial area as a result of the formation of pressure-enhanced $\mathrm{Al}_{2} \mathrm{O}_{3}$ /ionic liquid interactions under high pressures.
\end{abstract}

Keywords: imidazolium-based ionic liquids; infrared (IR); spectroscopy; high pressures

\section{Introduction}

Metal oxide nanoparticles possess different physicochemical properties in comparison to bulk materials, and the size of nanoparticles is responsible for the changes in their characteristics. Aluminum oxide, commonly called alumina, has tremendous applications in ceramics, medical products, and catalysis, to name a few [1-6]. Despite the expanding number of applications for aluminum oxide, the physical arrangement and interactions of liquids at $\mathrm{Al}_{2} \mathrm{O}_{3}$ surfaces remains poorly understood. Many experimental and theoretical studies have indicated the inherent complexity of interfacial interactions among certain liquid $/ \mathrm{Al}_{2} \mathrm{O}_{3}$ mixtures, and the immobilization of liquids on $\mathrm{Al}_{2} \mathrm{O}_{3}$ surfaces may improve the applicability of these materials in industry [4-6].

Ionic liquids (ILs) are a subset of low-melting-point salts that are known for high ionic conductivities, low vapor pressures, and low flammability. This combination of properties has opened opportunities for ionic liquids to serve as alternatives for conventional volatile organic solvents [7-10]. Although ILs offer advantages over conventional solvents, there is still relatively little information available on the structures of ILs in the bulk and along various interfaces $[7,9]$. The most extensively studied ILs are 1-alkyl-3-methylimidazolium salts, which feature asymmetric cations [7-10]. The imidazolium ions are usually paired with anions that have highly delocalized 
charges or are intrinsically bulky. The liquid structures of 1-alkyl-3-methylimidazolium salts are strongly affected by the long-range charge organization of the constituent ions and may be described in terms of an extended quasi-lattice, in which ions are viewed as occupying sites in a disorganized crystalline lattice $[11,12]$. In this model, any particular ion may be viewed as being trapped within a "cage" that is defined by its nearest neighbors. For ILs, the immediate solvation shell of an ion is most likely to be populated with counterions. Thus, an ion will have frequent opportunities to form short-lived associated species (e.g., ionic pairs, clusters, or aggregates) with the ions composing its cage $[7,13]$. With three hydrogen atoms bound to the imidazolium ring, the most acidic proton $\left(\mathrm{C}^{2}-\mathrm{H}\right)$ may form cation/anion hydrogen bonds. The nonpolar alkyl side chain of the cation provides another source of mesoscopic self-assembly of imidazolium ILs into nanostructured polar/nonpolar domains. Such nano-segregation is frequently observed in small angle X-ray scattering experiments for alkyl side chains containing four or more carbon atoms [14]. Various studies demonstrate significant heterogeneities in certain ionic liquid dynamics, and much of the heterogeneity may arise from nano-structured associations with polar and nonpolar regions [7-10,13,14].

Microscopic structures of certain ILs at solid surfaces have received considerable attention in recent years $[7,9,13]$. Nevertheless, the interfacial interactions caused by ionic liquid adsorption on the surface of $\mathrm{Al}_{2} \mathrm{O}_{3}$ are complex and depend on the molecular structures of ILs. It has been suggested that Coulombic forces, hydrogen bonding, and $\pi$ interactions may each play significant roles in defining the molecular ordering of ILs along the surface [7,9]. In this study, 1-butyl-3-methylimidazolium trifluoromethanesulfonate [BMIM][TFS] and nano- $\mathrm{Al}_{2} \mathrm{O}_{3}$ were chosen as a model IL and solid support, respectively. As pointed out by Andanson et al. [4], the TFS anion has many strong bands in the infrared spectrum. Unlike the bis(trifluoromethylsulfonyl)amide $\left(\mathrm{NTf}_{2}{ }^{-}\right)$anion, the TFS anion has only one conformer, which simplifies the spectral analysis. Nano- $\mathrm{Al}_{2} \mathrm{O}_{3}$ was chosen in this study mainly due to the wide application of nano- $\mathrm{Al}_{2} \mathrm{O}_{3}$, particularly in the field of catalysis.

Vibrational spectroscopy is particularly useful in studying ionic liquid $/ \mathrm{Al}_{2} \mathrm{O}_{3}$ interactions because the frequencies and intensities of cation and anion vibrational modes are sensitive to their immediate potential energy environments. In this way, changes in band frequencies and intensities may be used to infer changes in the local structures of the ions. Many spectroscopic studies on ionic liquid interactions with nanostructured metal oxides focus on changing the temperature of the composite or varying the molecular structure of the ionic liquid in contact with the solid (e.g., lengthening the alkyl side chain) to probe the interfacial interactions [1-9]. Hydrostatic pressure is another degree of freedom that can continuously tune the structures, interactions, and solvation of chemical systems. In the case of temperature changes, the sample's properties can change significantly due to a simultaneous change in thermal energy and volume. However, the use of pressure as a variable allows one to separate the thermal and volume effects. Studies have shown the potential significance that pressure has on controlling the strength of intermolecular hydrogen bonding, especially the relatively weak $\mathrm{C}-\mathrm{H}-\mathrm{X}$ hydrogen bonds, where $X$ is an anion atom [15-18]. Pressure-dependent changes in spectral features indicate that nanoparticles tend to influence local structures of certain ILs under high pressures. As certain ILs have been employed as graphene stabilizers, previous studies suggest that imidazolium ILs with short alkyl chain lengths $(n<4)$ may be suitable choices to modulate the performance of energy storage devices via pressure-enhanced interfacial interactions [15]. In order to obtain detailed insights into the ionic liquid $/ \mathrm{Al}_{2} \mathrm{O}_{3}$ interactions, we use variable pressure as a window into the nature of hydrogen bonding structures of imidazolium ionic liquid $/ \mathrm{Al}_{2} \mathrm{O}_{3}$ mixtures in this article.

\section{Materials and Methods}

Samples were prepared using 1-butyl-3-methylimidazolium trifluoromethanesulfonate ([BMIM][TFS], $>95 \%$, Fluka, Morris Plains, NJ, USA), and nanosized-aluminum oxide (nano- $\mathrm{Al}_{2} \mathrm{O}_{3}$, $<50 \mathrm{~nm}$, Aldrich, St. Louis, MO, USA). The nano- $\mathrm{Al}_{2} \mathrm{O}_{3}$ powders (ca. $0.006 \mathrm{~g}$ ) were mixed with [BMIM][TFS] (ca. $0.06 \mathrm{~g}$ ), followed by sonication for $30 \mathrm{~min}$, centrifugation, several washes of the precipitate with ethanol ( $0.1 \mathrm{~mL}$ for a single wash), centrifugation, and drying under vacuum for 
$3 \mathrm{~h}$. The infrared spectra of samples measured at ambient pressure were taken by filling samples in a cell characterized by two $\mathrm{CaF}_{2}$ windows without the spacers. A diamond anvil cell (DAC) of Merrill-Bassett design with a diamond culet size of $0.6 \mathrm{~mm}$ was used to generate pressures of up to ca. 2 GPa. Two type-IIa diamonds were used for mid-infrared measurements. Infrared (IR) spectra of the samples were measured on a Fourier transform spectrophotometer (Spectrum RXI, Perkin-Elmer, Naperville, IL, USA) equipped with an LITA (lithium tantalite) mid-infrared detector. The infrared beam was condensed through a $5 \times$ beam condenser onto the sample in the diamond anvil cell. To remove the absorption of the diamond anvils, the absorption spectra of the DAC were measured first and subtracted from those of the samples. Samples were contained in a 0.3-mm-diameter hole in a $0.25-\mathrm{mm}$-thick Inconel gasket mounted on the DAC. To reduce the absorbance of the samples, $\mathrm{CaF}_{2}$ crystals (prepared from a $\mathrm{CaF}_{2}$ optical window) were placed into the holes and compressed to be transparent prior to inserting the samples. A sample droplet filled the empty space of the entire hole of the gasket in the DAC, which was subsequently sealed when the opposing anvils were pushed toward one another. Typically, we chose a resolution of $4 \mathrm{~cm}^{-1}$ (data point resolution of $2 \mathrm{~cm}^{-1}$ ). For each spectrum, 1000 scans were compiled. Pressure calibration was performed following Wong's method [19,20].

\section{Results and Discussion}

Figure 1 shows the infrared spectra of pure [BMIM][TFS], the [BMIM][TFS] $/ \mathrm{Al}_{2} \mathrm{O}_{3}$ mixture, the mixture after two washes with ethanol, and the mixture after five washes obtained under ambient pressure. The amount of [BMIM][TFS] are adjusted by controlling the numbers of washes in this study; the amount of [BMIM][TFS] becomes almost depleted after five washes, as shown in Figure 1d. The IR spectrum of pure [BMIM][TFS] in Figure 1a shows two imidazolium C-H bands at 3116 and $3155 \mathrm{~cm}^{-1}$, a shoulder to the $3155 \mathrm{~cm}^{-1}$ band at $3098 \mathrm{~cm}^{-1}$, and three alkyl C-H bands at 2877, 2940, and $2967 \mathrm{~cm}^{-1}$, respectively [21]. As shown in Figure 1, no appreciable changes in spectral features and band frequencies of $\mathrm{C}-\mathrm{H}$ vibrations occurred as [BMIM][TFS] was mixed with nano- $\mathrm{Al}_{2} \mathrm{O}_{3}$, except for a noticeable absorption caused by $\mathrm{O}-\mathrm{H}$ groups from the surfaces of nano- $\mathrm{Al}_{2} \mathrm{O}_{3}$ that appears in Figure $1 \mathrm{~d}$. These results indicate that IR measurements recorded under ambient pressure may not be sensitive enough to monitor the interfacial interactions between the $\mathrm{BMIM}^{+}$cation and nano- $\mathrm{Al}_{2} \mathrm{O}_{3}$.

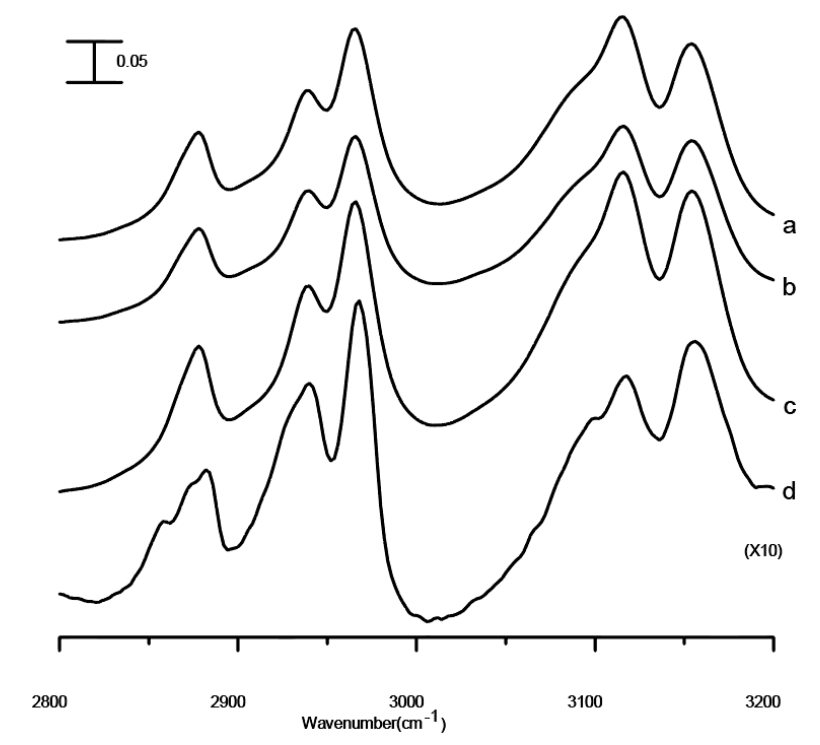

Figure 1. Infrared (IR) spectra of pure 1-butyl-3-methylimidazolium trifluoromethanesulfonate ([BMIM][TFS]) (curve a), the [BMIM][TFS] $/ \mathrm{Al}_{2} \mathrm{O}_{3}$ mixture (curve $\mathrm{b}$ ), the mixture after two washes with ethanol (curve c), and the mixture after five washes (curve d), recorded under ambient pressure. Curve $d$ was baseline-corrected by subtracting a straight line. Relative intensities of the IR bands are given in increments of 0.05 ; the scale bar is displayed in the upper left corner of the figure. 
Figure 2 displays the IR spectra of samples in the spectral range between 1000 and $1350 \mathrm{~cm}^{-1}$. This region of the spectrum is predominately dominated by absorption bands due to vibrations of the TFS ${ }^{-}$anions. The bands at ca. 1165 and $1226 \mathrm{~cm}^{-1}$ are assigned to $\mathrm{CF}_{3}$ asymmetric and symmetric stretching vibrations, respectively. The absorption bands located near 1032 and $1270 \mathrm{~cm}^{-1}$ (doublet) are assigned to the symmetric and asymmetric stretching vibration of the $\mathrm{SO}_{3}$ group of the TFS ${ }^{-}$ anion, respectively. Comparing the spectral features of Figure $2 \mathrm{~d}$ with those of Figure 2a reveal mild frequency shifts and narrowing for the bands at ca. 1165 and $1270 \mathrm{~cm}^{-1}$, caused by the presence of nano- $\mathrm{Al}_{2} \mathrm{O}_{3}$. The absorption of $\mathrm{CaF}_{2}$ windows (below $1050 \mathrm{~cm}^{-1}$ ) also becomes obvious in Figure $2 \mathrm{~d}$.

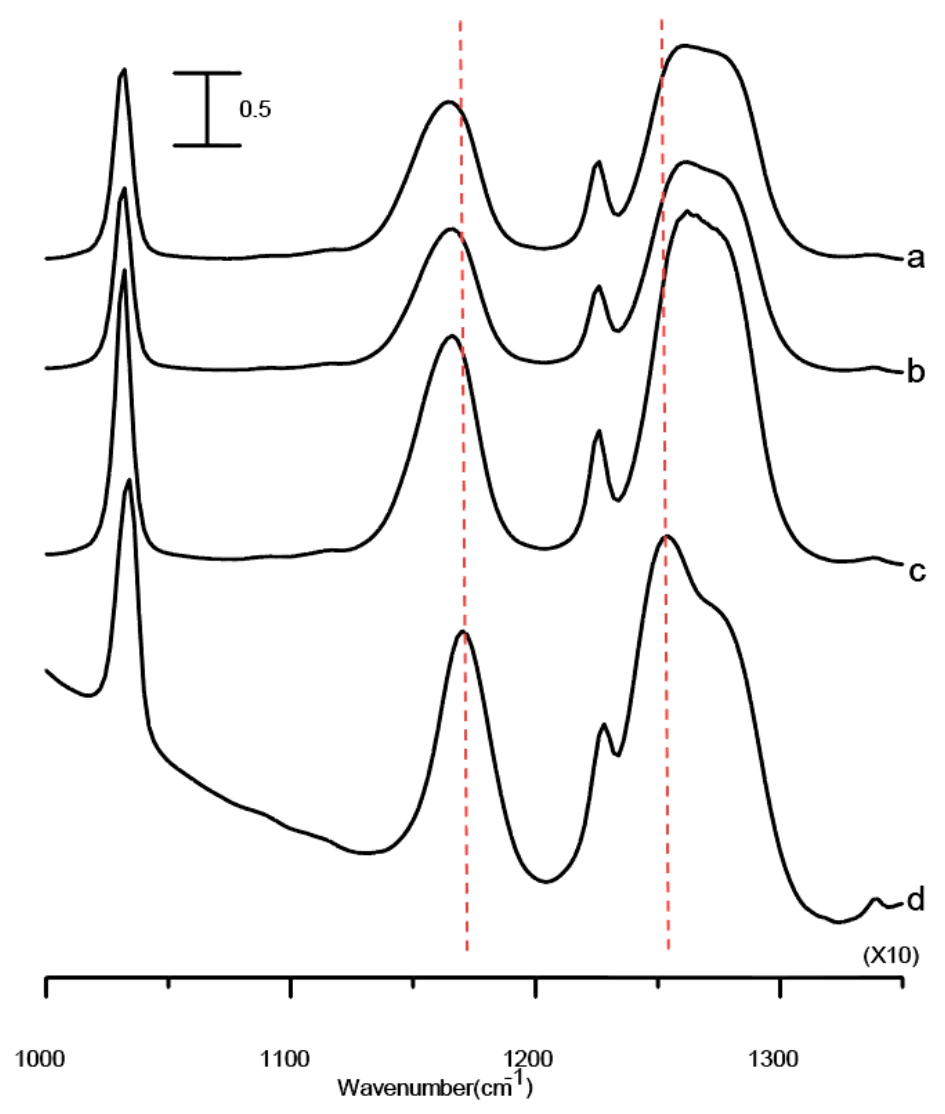

Figure 2. IR spectra of pure [BMIM][TFS] (curve a), the [BMIM][TFS] $/ \mathrm{Al}_{2} \mathrm{O}_{3}$ mixture (curve b), the mixture after two washes with ethanol (curve c), and the mixture after five washes (curve d), recorded under ambient pressure. Relative intensities of the IR bands are given in increments of 0.05; the scale bar is displayed in the upper left corner of the figure.

Figure 3 displays the IR spectra of a sample from a [BMIM][TFS] $/ \mathrm{Al}_{2} \mathrm{O}_{3}$ mixture obtained under ambient pressure (curve a) and at 0.4 (curve b), 0.7 (curve c), 1.1 (curve d), 1.5 (curve e), 1.8 (curve f), and 2.5 GPa (curve g). The elevation of pressure to 0.4 GPa leads to a phase transition, and the imidazolium C-H bands are blue-shifted to 3102,3132 , and $3168 \mathrm{~cm}^{-1}$ in Figure $3 \mathrm{~b}$. The sharper structures of imidazolium C-H bands revealed in Figure $3 \mathrm{~b}-\mathrm{g}$ are in part due to the anisotropic environment in a solid structure. We note that spectral features of the [BMIM][TFS] $/ \mathrm{Al}_{2} \mathrm{O}_{3}$ mixture in Figure 3 are almost identical to those of pure [BMIM][TFS], as revealed in our previous studies [21]. The results of Figure 3 indicate that the presence of nano- $\mathrm{Al}_{2} \mathrm{O}_{3}$ does not perturb ion/ion interactions from the perspective of the $\mathrm{C}-\mathrm{H}$ stretching motions of the cations. Instead, the blue-shifts revealed in Figure 3 may originate from the combined effect of the overlap repulsion enhanced by hydrostatic pressure, $\mathrm{C}-\mathrm{H}-\mathrm{O}$ contacts, and so forth. The increased understanding of weak interactions, such as hydrogen bonding between the $\mathrm{C}-\mathrm{H}$ groups on the imidazolium cation and oxygen atoms of the 
TFS $^{-}$anion, has required an expansion of the classical definition of hydrogen bonding. One of the intriguing aspects of $\mathrm{C}-\mathrm{H}-\mathrm{O}$ interactions is that the $\mathrm{C}-\mathrm{H}$ bond tends to shorten as a result of the formation of blue-shifting hydrogen bonds. We demonstrate in Figure 3 that high pressure is a valuable method to probe pressure-enhanced blue-shifting of $\mathrm{C}-\mathrm{H}$ hydrogen bonds in the [BMIM][TFS] $/ \mathrm{Al}_{2} \mathrm{O}_{3}$ mixture. Scheiner's group and Dannenberg's group indicated that the origin of both red-shifting and blue-shifting hydrogen bonds may be the same, and suggested that both types of hydrogen bonds result from a combination of electrostatic, polarization, charge transfer, dispersion, and exchange/steric repulsion forces between proton donors and acceptors [22,23].

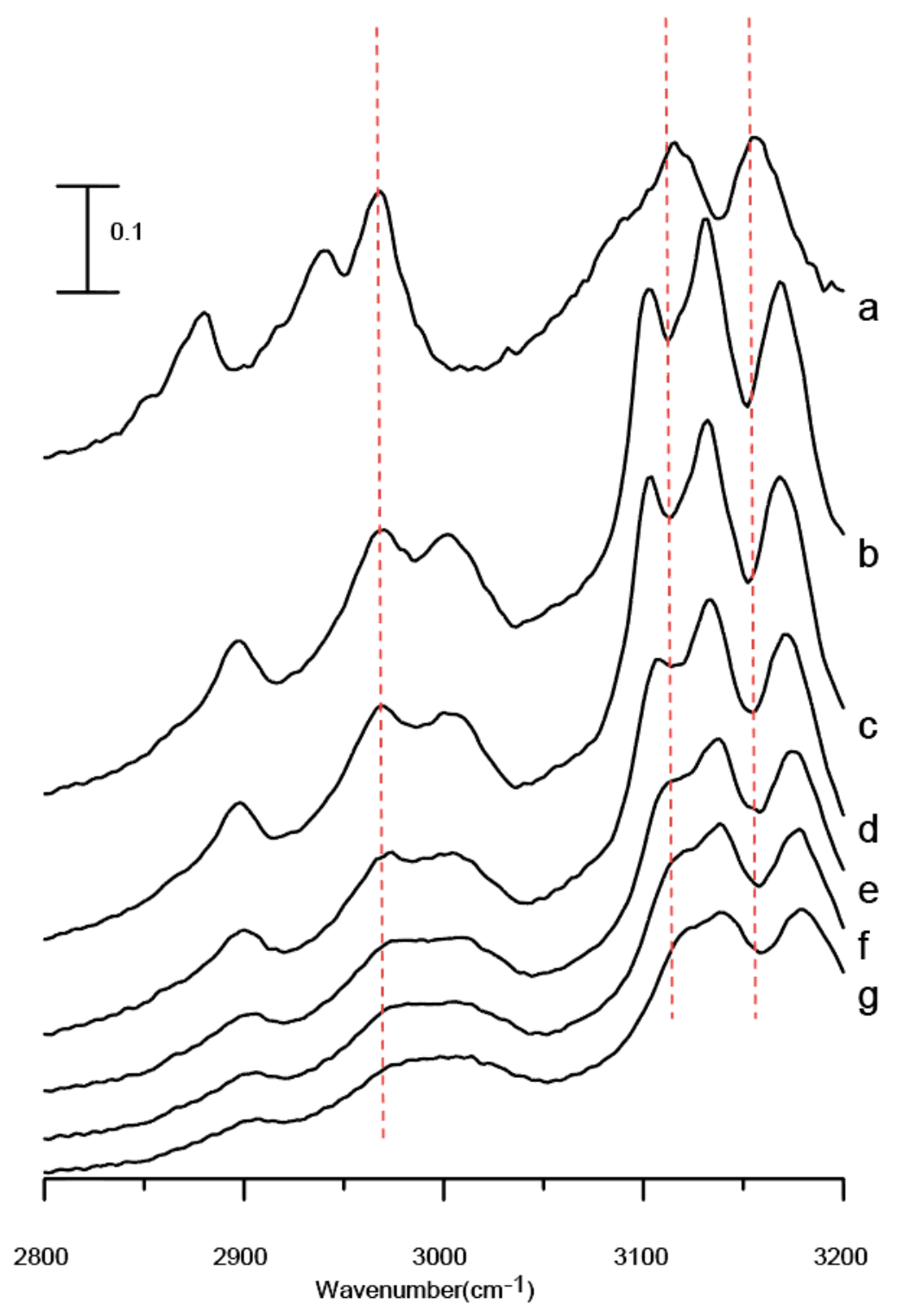

Figure 3. IR spectra of the $[\mathrm{BMIM}][\mathrm{TFS}] / \mathrm{Al}_{2} \mathrm{O}_{3}$ mixture obtained under ambient pressure (curve a) and at 0.4 (curve b), 0.7 (curve c), 1.1 (curve d), 1.5 (curve e), 1.8 (curve f), and 2.5 GPa (curve g). Relative intensities of the IR bands are given in increments of 0.1 ; the scale bar is displayed in the upper left corner of the figure.

Figure 4 shows the infrared spectra of the [BMIM][TFS]/ $\mathrm{Al}_{2} \mathrm{O}_{3}$ mixture attributed to the absorption of TFS $^{-}$anions under various pressures. As the mixture is compressed to $0.4 \mathrm{GPa}$ in Figure $4 \mathrm{~b}$, the $1270 \mathrm{~cm}^{-1}$ (doublet) band narrowed and the band at $1165 \mathrm{~cm}^{-1}$ splits into two 
distinct bands at ca. 1152 and $1175 \mathrm{~cm}^{-1}$. These spectral changes may indicate a pressure-induced phase transition arising from both structural reorganization (due to ordered local structures) and a decrease in inhomogeneous broadening. The spectral features of the [BMIM][TFS] $/ \mathrm{Al}_{2} \mathrm{O}_{3}$ mixture revealed in Figure 4 are almost identical to those of pure [BMIM][TFS]. In contrast to the bandwidth narrowing of asymmetric $\mathrm{SO}_{3}$ stretching vibration at ca. $1270 \mathrm{~cm}^{-1}$, the $1032 \mathrm{~cm}^{-1}$ band (symmetric $\mathrm{SO}_{3}$ stretching) displays anomalous bandwidth broadening upon compression in Figure $4 \mathrm{~b}-\mathrm{g}$. It is likely that transverse optic (TO)-longitudinal optic (LO) splitting of the symmetric $\mathrm{SO}_{3}$ stretching band plays a non-negligible role in Figure 4 [11,12,24].

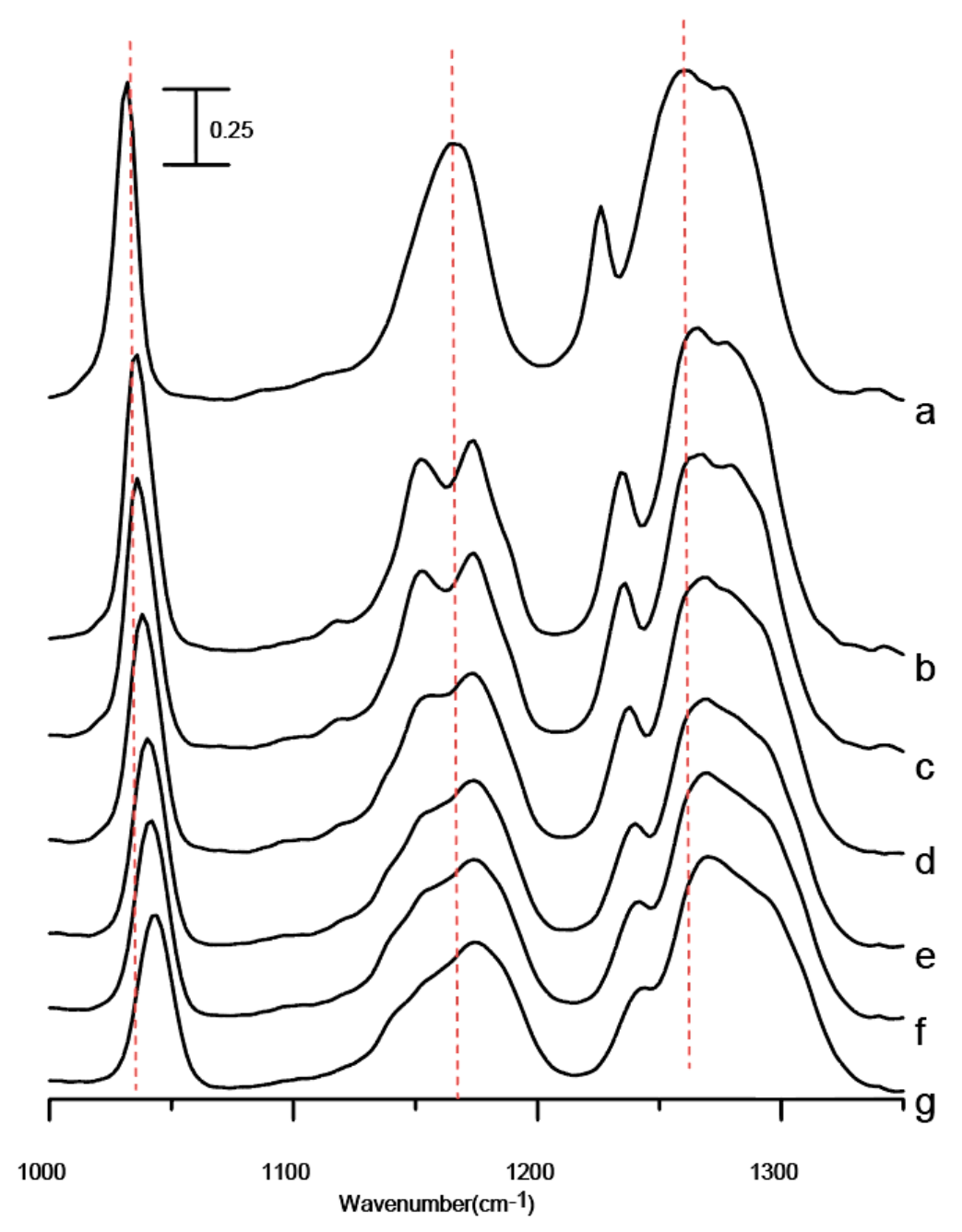

Figure 4. IR spectra of the [BMIM][TFS] $/ \mathrm{Al}_{2} \mathrm{O}_{3}$ mixture obtained under ambient pressure (curve a) and at 0.4 (curve b), 0.7 (curve c), 1.1 (curve d), 1.5 (curve e), 1.8 (curve f), and 2.5 GPa (curve g). Relative intensities of the IR bands are given in increments of 0.25 ; the scale bar is displayed in the upper left corner of the figure.

Figure 5 displays the IR spectra of a [BMIM][TFS] $/ \mathrm{Al}_{2} \mathrm{O}_{3}$ mixture after five washes with ethanol obtained under ambient pressure (curve a) and at 0.4 (curve b), 0.7 (curve c), 1.1 (curve d), 1.5 (curve e), 1.8 (curve f), and 2.5 GPa (curve g). As shown in Figure 5a,b, no appreciable changes in the spectral features of $\mathrm{C}-\mathrm{H}$ vibrations occur as the sample is compressed to $0.4 \mathrm{GPa}$. This result is remarkably different from that is revealed for the [BMIM][TFS] $/ \mathrm{Al}_{2} \mathrm{O}_{3}$ mixture in Figure $3 \mathrm{~b}$. It appears that the presence of nano- $\mathrm{Al}_{2} \mathrm{O}_{3}$ has an influence on the supramolecular assemblies of the remaining ILs on the surface of $\mathrm{Al}_{2} \mathrm{O}_{3}$ after five washes and under high pressures, as revealed in Figure 5. The nano- $\mathrm{Al}_{2} \mathrm{O}_{3}$ 
surfaces may weaken the cation/anion interactions in the interfacial area as a result of the formation of enhanced ionic liquid/particle surface interactions under high pressures. The $\mathrm{Al}_{2} \mathrm{O}_{3}$ /ionic liquid interactions may also affect the hydrogen-bonded network of interfacial [BMIM][TFS] under high pressures. In contrast to the results obtained under ambient pressure (Figure 1), the local structures of both the imidazolium $\mathrm{C}-\mathrm{H}$ and alkyl $\mathrm{C}-\mathrm{H}$ groups are disturbed under high pressures for the mixture after five washes, shown in Figure 5, when compared to the pressure dependence of the [BMIM][TFS] $/ \mathrm{Al}_{2} \mathrm{O}_{3}$ mixture (Figure 3). Therefore, high pressures may have the potential to control the order and strength of ionic liquid/surface interactions in this system.

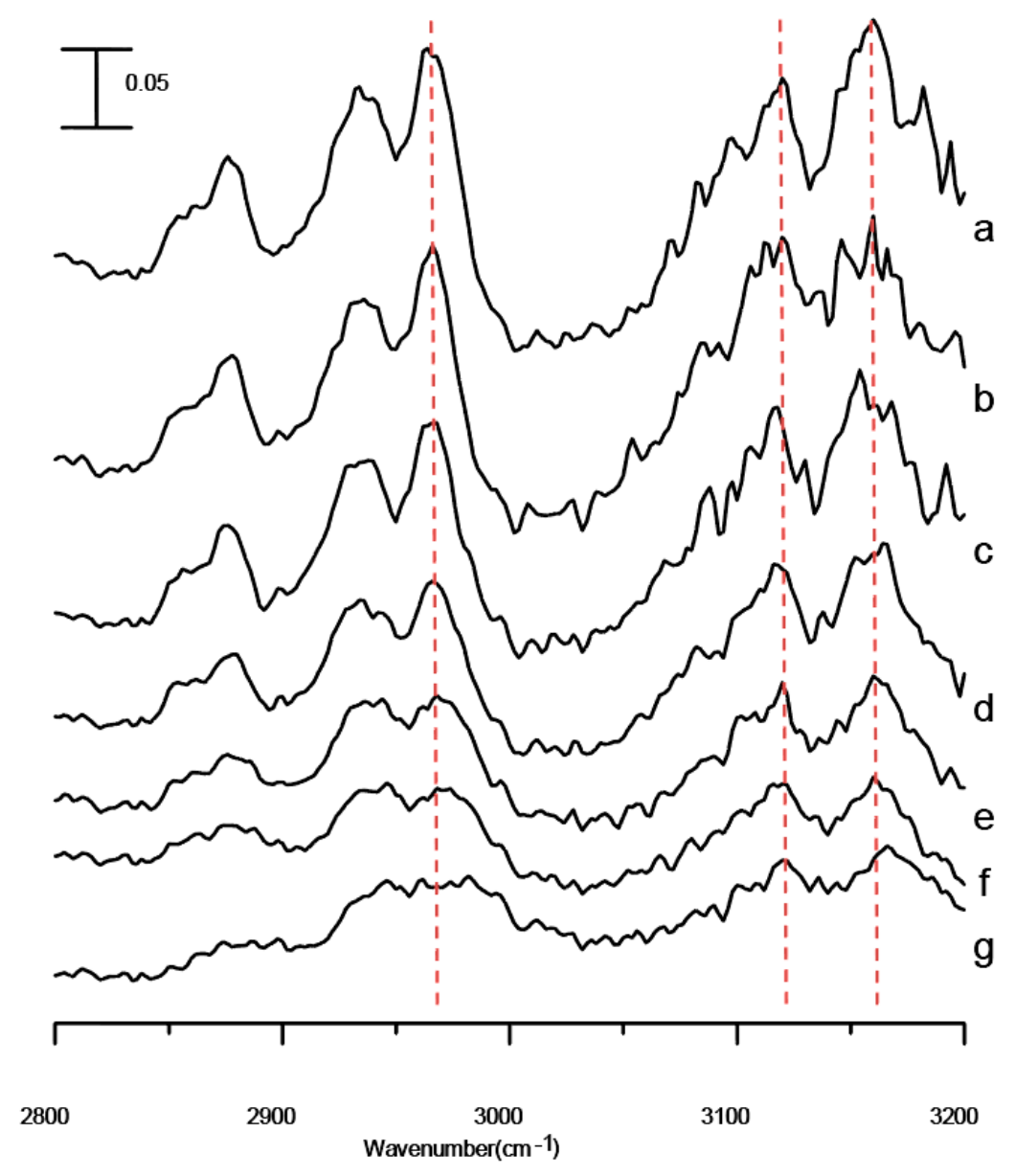

Figure 5. IR spectra of the mixture after five washes with ethanol obtained under ambient pressure (curve a) and at 0.4 (curve b), 0.7 (curve c), 1.1 (curve d), 1.5 (curve e), 1.8 (curve f), and 2.5 GPa (curve g). All curves were baseline-corrected by subtracting straight lines. Relative intensities of the IR bands are given in increments of 0.05 ; the scale bar is displayed in the upper left corner of the figure.

Figure 6 shows the infrared spectra of the mixture after five washes in the spectral range between 1000 and $1350 \mathrm{~cm}^{-1}$ obtained under ambient pressure (curve a) and at 0.4 (curve b), 0.7 (curve c), 1.1 (curve d), 1.5 (curve e), 1.8 (curve f), and 2.5 GPa (curve g). The absence of drastic band-narrowing and splitting in Figure 6 suggests that interfacial ionic liquid $/ \mathrm{Al}_{2} \mathrm{O}_{3}$ interactions of the mixture after five washes may be enhanced by the application of pressure. As revealed in Figure $6 \mathrm{~g}$, the absorption bands display mild blue-shifts in frequency as the mixture is compressed to $2.5 \mathrm{GPa}$. 


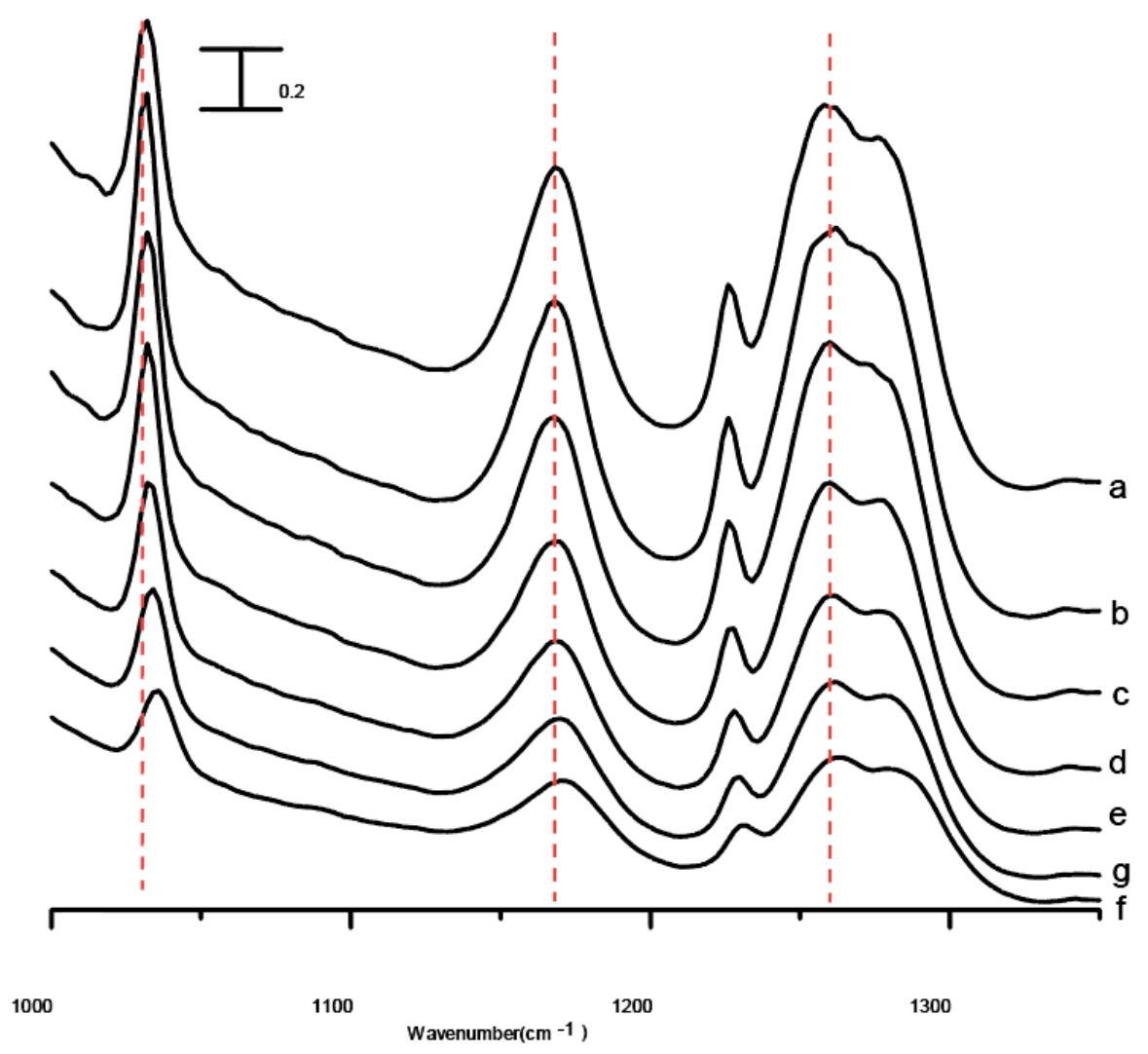

Figure 6. IR spectra of the mixture after five washes with ethanol obtained under ambient pressure (curve a) and at 0.4 (curve b), 0.7 (curve c), 1.1 (curve d), 1.5 (curve e), 1.8 (curve f), and $2.5 \mathrm{GPa}$ (curve g). Relative intensities of the IR bands are given in increments of 0.2; the scale bar is displayed in the upper left corner of the figure.

To illustrate the frequency shift, the pressure dependence of $\mathrm{C}-\mathrm{H}$ stretching frequencies of the [BMIM][TFS] $/ \mathrm{Al}_{2} \mathrm{O}_{3}$ mixture, the mixture after two washes with ethanol, and the mixture after five washes is plotted in Figure 7. The $\mathrm{C}-\mathrm{H}$ bands corresponding to the [BMIM][TFS] $/ \mathrm{Al}_{2} \mathrm{O}_{3}$ mixture display significant blue-shifts in frequency as the pressure increases to $0.4 \mathrm{GPa}$, and then undergo mild shifts in frequency when the pressure increases from 0.4 to $2.5 \mathrm{GPa}$. The discontinuous jump occurring around $0.4 \mathrm{GPa}$ becomes less obvious for the mixture after two washes with ethanol. In Figure 7, we observe no discontinuous jump in frequency for the mixture after five washes. It is known that the hydrogen bond cooperativity due to concerted charge transfer can greatly enhance the strength of the individual hydrogen bonds involved in the coupling [25]. Hydrogen-bonding non-additivity and the size of clusters are suggested to be responsible for the enhancement of hydrogen bonding [25,26]. Thus, the anomalous jump in frequency at $0.4 \mathrm{GPa}$ can be attributed to both the cooperative and geometric effects of blue-shifting hydrogen bonds. The disappearance of the discontinuous jump upon the reduction of ionic liquid through washing with ethanol may indicate the decrease in strength of blue-shifting hydrogen bonding as $\mathrm{C}-\mathrm{H} /$ anion interactions are replaced by $\mathrm{C}-\mathrm{H} / \mathrm{Al}_{2} \mathrm{O}_{3}$ interactions. The loss of the frequency jump at $0.4 \mathrm{GPa}$ due to washing is related to a reduction of the amount of ionic liquid along the interface. At thicker values, there is likely enough IL to form crystallites. If there is just a monolayer (or about that), crystallization might be difficult and hydrogen bond cooperativity may be reduced. 

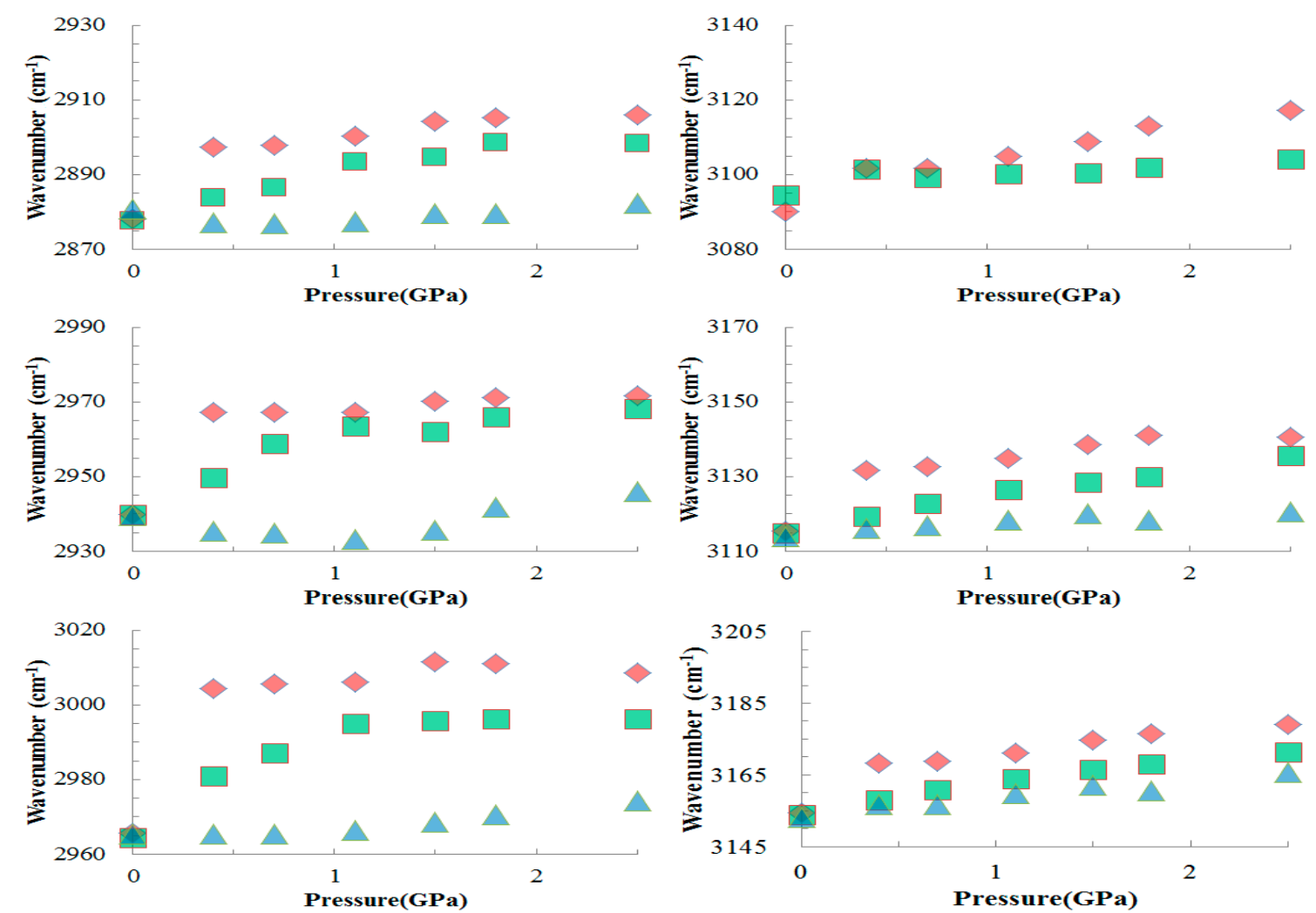

Figure 7. Pressure dependence of the $\mathrm{C}-\mathrm{H}$ stretching frequencies of the $[\mathrm{BMIM}][\mathrm{TFS}] / \mathrm{Al}_{2} \mathrm{O}_{3}$ mixture (diamonds), the mixture after two washes with ethanol (squares), and the mixture after five washes (triangles).

This study indicates that high pressures can serve as a useful tool to probe the strength of blue-shifting $\mathrm{C}-\mathrm{H}$ hydrogen bonding in ILs deposited along the surface of nano- $\mathrm{Al}_{2} \mathrm{O}_{3}$ surfaces. Furthermore, the surfaces of $\mathrm{Al}_{2} \mathrm{O}_{3}$ appear to be capable of breaking or weakening the interactions of the cation/anion clusters, presumably in favor of stable ionic liquid $/ \mathrm{Al}_{2} \mathrm{O}_{3}$ interactions under high pressures. Supplementary data (IR spectra of the mixture after two washes and pure [BMIM][TFS] with 99\% purity) are included in Supplementary Materials (see Figures S1-S3).

\section{Conclusions}

In this study, we demonstrate that the effect of nano- $\mathrm{Al}_{2} \mathrm{O}_{3}$ on hydrogen bonding networks of [BMIM][TFS] can be probed by high-pressure infrared spectroscopy. There are no remarkable changes in the spectral features of [BMIM][TFS] in the presence of nano- $\mathrm{Al}_{2} \mathrm{O}_{3}$ at ambient pressure. Nevertheless, nano- $\mathrm{Al}_{2} \mathrm{O}_{3}$ may perturb the cation/anion aggregations as a result of the formation of pressure-enhanced ionic liquid $/ \mathrm{Al}_{2} \mathrm{O}_{3}$ interactions when high pressure is applied to the composite materials. The relative strength of blue-shifting $\mathrm{C}-\mathrm{H}$ hydrogen bonding, i.e., [BMIM][TFS] $>$ [BMIM] $-\mathrm{Al}_{2} \mathrm{O}_{3}$, is also investigated by varying the applied pressure. This study indicates that high pressures may have the potential to tune the strength of ionic liquid $/ \mathrm{Al}_{2} \mathrm{O}_{3}$ surface interactions. Moreover, high-pressure infrared spectroscopy provides an alternative avenue for a better understanding the interfacial properties of ILs in contact with nanostructured metal oxides.

Supplementary Materials: The following are available online at http:/ /www.mdpi.com/2076-3417/7/8/855/s1, Figure S1: IR spectra of the mixture after two washes with ethanol obtained under ambient pressure (curve a) and at 0.4 (curve b), 0.7 (curve c), 1.1 (curve d), 1.5 (curve e), 1.8 (curve f), and 2.5 GPa (curve g), Figure S2: Infrared spectra of the mixture after two washes with ethanol obtained under ambient pressure (curve a) and at 0.4 (curve b), 0.7 (curve c), 1.1 (curve d), 1.5 (curve e), 1.8 (curve f), and 2.5 GPa (curve g), Figure S3: Infrared 
spectra of pure [BMIM][TFS] (99\%, Lot: N00364.7, UniRegion) obtained under ambient pressure (curve a) and at 0.4 (curve b), 0.7 (curve c), 1.1 (curve d), 1.5 (curve e), 1.8 (curve f), and 2.3 GPa (curve g).

Acknowledgments: The authors thank the National Dong Hwa University and Ministry of Science and Technology (Contract No. MOST 106-2113-M-259-002) of Taiwan for financial support. The authors thank Yen-Hsu Chang and Min-Hsiu Shen for the assistance.

Author Contributions: Hai-Chou Chang is the idea source, writer of the manuscript, and provided data interpretation. Teng-Hui Wang contributed to FTIR spectra measurements. Christopher M. Burba is the co-writer and provided the idea of TO-LO splitting.

Conflicts of Interest: The authors declare no conflict of interest.

\section{References}

1. Babucci, M.; Akcay, A.; Balci, V.; Uzan, A. Thermal stability of limits of imidazolium ionic liquids immobilized on metal oxides. Langmuir 2015, 31, 9163-9176. [CrossRef] [PubMed]

2. Lee, H.J.; Park, J.H. Effect of hydrophobic modification on carbon dioxide absorption using porous alumina $\left(\mathrm{Al}_{2} \mathrm{O}_{3}\right)$ hollow fiber membrane contactor. J. Membr. Sci. 2016, 518, 79-87. [CrossRef]

3. Pizzozzaro, M.A.; Drobek, M.; Petit, E.; Guerrero, G.; Hesemann, P.; Julbe, A. Design of phosphonated imidazolium-based ionic liquids grafted on $\gamma$-alumina: Potential model for hybrid membranes. Int. J. Mol. Sci. 2016, 17, 1212. [CrossRef] [PubMed]

4. Andanson, J.M.; Baiker, A. Interactions of 1-ethyl-3-methylimidazolium trifluoromethanesulfonate ionic liquid with alumina nanoparticles and organic solvents studied by infrared spectroscopy. J. Phys. Chem. C 2013, 117, 12210-12217. [CrossRef]

5. Schernich, S.; Kostyshyn, D.; Wagner, V.; Taccardi, N.; Laurin, M.; Wasserscheid, P.; Libuda, J. Interactions between the room-temperature ionic liquid $\left[\mathrm{C}_{2} \mathrm{C}_{1} \mathrm{Im}\right][\mathrm{OTf}]$ and $\mathrm{Pd}(111)$, well-ordered $\mathrm{Al}_{2} \mathrm{O}_{3}$, and supported Pd model catalysts from IR spectroscopy. J. Phys. Chem. C 2014, 118, 3188-3193. [CrossRef]

6. Fuentes, C.E.S.; Guzman-Lucero, D.; Torres-Rodriguez, M.; Likhanoca, N.V.; Bolanos, J.N.; Loivares-Xometl, O.; Lijanova, I.V. $\mathrm{CO}_{2} / \mathrm{N}_{2}$ separation using alumina supported membranes based on new functionalized ionic liquids. Sep. Purif. Technol. 2017, 182, 59-68. [CrossRef]

7. Zhang, S.; Zhang, J.; Zhang, Y.; Deng, Y. Nanoconfined ionic liquids. Chem. Rev. 2017, 117, 6755-6833. [CrossRef] [PubMed]

8. Singh, D.K.; Rathke, B.; Kiefer, J.; Materny, A. Molecular structure and interactions in the ionic liquid 1-ethyl-3-methylimidazolium trifluoromethanesulfonate. J. Phys. Chem. A 2016, 120, 6274-6286. [CrossRef] [PubMed]

9. Fedorov, M.V.; Kornyshev, A.A. Ionic liquids at electrified interfaces. Chem. Rev. 2014, 114, $2978-3036$. [CrossRef] [PubMed]

10. Wulf, A.; Fumino, K.; Michalik, D.; Ludwig, R. IR and NMR properties of ionic liquids: Do they tell us the same thing? ChemPhysChem 2007, 8, 2265-2269. [CrossRef] [PubMed]

11. Burba, C.M.; Janzen, J.; Butson, E.D.; Coltrain, G.L. Using FT-IR spectroscopy to measure charge organization in ionic liquids. J. Phys. Chem. B 2013, 117, 8814-8820. [CrossRef] [PubMed]

12. Burba, C.M.; Janzen, J.; Butson, E.D.; Coltrain, G.L. Correction to "using FT-IR spectroscopy to measure charge organization in ionic liquids". J. Phys. Chem. B 2016, 120, 3591-3592. [CrossRef] [PubMed]

13. Chang, H.C.; Hung, T.C.; Wang, H.S.; Chen, T.Y. Local structures of ionic liquids in the presence of gold under high pressures. AIP Adv. 2013, 3, 032147. [CrossRef]

14. Triolo, A.; Russina, O.; Bleif, H.J. Nanoscale segregation in room temperature ionic liquids. J. Phys. Chem. $B$ 2007, 111, 4641-4644. [CrossRef] [PubMed]

15. Chang, H.C.; Hsu, D.T. Interactions of ionic liquids and surfaces of graphene related nanoparticles under high pressures. Phys. Chem. Chem. Phys. 2017, 19, 12269-12275. [CrossRef] [PubMed]

16. Chang, H.C.; Zhang, R.L.; Hsu, D.T. The effect of pressure on cation-cellulose interactions in cellulose/ionic liquid mixtures. Phys. Chem. Chem. Phys. 2015, 17, 27573-27578. [CrossRef] [PubMed]

17. Chang, H.C.; Jiang, J.C.; Kuo, M.H.; Hsu, D.T.; Lin, S.H. Pressure-enhanced surface interactions between nano- $\mathrm{TiO}_{2}$ and ionic liquid mixtures probed by high pressure IR spectroscopy. Phys. Chem. Chem. Phys. 2015, 17, 21143-21148. [CrossRef] [PubMed] 
18. Chang, H.C.; Tsai, T.T.; Kuo, M.H. Using high-pressure infrared spectroscopy to study the interactions between triblock copolymers and ionic liquids. Macromolecules 2014, 47, 3052-3058. [CrossRef]

19. Wong, P.T.T.; Moffatt, D.J. The uncoupled O-H or O-D stretch in water as an internal pressure gauge for high-pressure infrared spectroscopy of aqueous systems. Appl. Spectrosc. 1987, 41, 1070-1072. [CrossRef]

20. Wong, P.T.T.; Moffatt, D.J.; Baudias, F.L. Crystalline quartz as an internal pressure calibrant for high-pressure infrared spectroscopy. Appl. Spectrosc. 1985, 39, 733-735. [CrossRef]

21. Chang, H.C.; Chang, S.C.; Hung, T.C.; Jiang, J.C.; Kuo, J.L.; Lin, S.H. A high-pressure study of the effects of $\mathrm{TiO}_{2}$ nanoparticles on the structural organization of ionic liquids. J. Phys. Chem. C 2011, 115, 23778-23783. [CrossRef]

22. Gu, Y.L.; Kar, T.; Scheiner, S. Fundamental properties of the C-H-O interactions: Is it a true hydrogen bond? J. Am. Chem. Soc. 1999, 121, 9411-9422. [CrossRef]

23. Masunov, A.; Dannenberg, J.J.; Contreras, R.W. C-H bond-shortening upon hydrogen bond formation: Influence of an electric field. J. Phys. Chem. A 2001, 105, 4737-4740. [CrossRef]

24. Burba, C.M.; Frech, R. Existence of optical phonons in the room temperature ionic liquid 1-ethyl-3methylimidazolium trifluoromethanesulfonate. J. Chem. Phys. 2011, 134, 134503. [CrossRef] [PubMed]

25. Knorr, A.; Stange, P.; Fumino, K.; Weinhold, F.; Ludwig, R. Spectroscopic evidence for clusters of like-charged ions in ionic liquids stabilized by cooperative hydrogen bonding. ChemPhysChem 2016, 17, 458-462.

26. Roth, C.; Appelhagen, A.; Jobst, N.; Ludwig, R. Microheterogeneities in ionic-liquid-methanol solutions studied by FTIR spectroscopy, DFT calculations and molecular dynamics simulations. ChemPhysChem 2012, 13, 1708-1717. [CrossRef] [PubMed]

(c) 2017 by the authors. Licensee MDPI, Basel, Switzerland. This article is an open access article distributed under the terms and conditions of the Creative Commons Attribution (CC BY) license (http://creativecommons.org/licenses/by/4.0/). 